\title{
Effect of grain boundary on resistive magnetodielectric property of polycrystalline $\gamma-\mathrm{Fe}_{2} \mathrm{O}_{3}$
}

\author{
T. Bonaedy $\cdot$ Y.S. Koo $\cdot$ K.D. Sung $\cdot$ K.M. Song $\cdot$ \\ N. Hur · J.H. Jung
}

Received: 14 March 2008 / Accepted: 16 June 2008 / Published online: 6 July 2008

(c) Springer-Verlag 2008

\begin{abstract}
We have investigated the role of the grain boundary on the resistive magnetodielectric property of polycrystalline $\gamma-\mathrm{Fe}_{2} \mathrm{O}_{3}$ through impedance spectroscopy measurements. Depending on the sample preparation temperature, the dielectric constant of $\gamma-\mathrm{Fe}_{2} \mathrm{O}_{3}$ is significantly different especially at low frequencies $\left(<10^{4} \mathrm{~Hz}\right)$ and high temperatures $(>200 \mathrm{~K})$. The value of the magnetodielectric effect at a specific frequency and the resonance frequency for the maximized magnetodielectric effect are different, although polycrystalline $\gamma-\mathrm{Fe}_{2} \mathrm{O}_{3}$ samples show a quite similar magnetoresistance. Through the experimentally obtained resistance ratio between the grain and the grain boundary, we can reproduce the magnetodielectric curves based on the Maxwell-Wagner model and the measured magnetoresistance.
\end{abstract}

PACS 75.50.Gg $\cdot 75.80 .+q \cdot 77.22 . \mathrm{Ch}$

\section{Introduction}

The control of electrical properties of multiferroic materials, where more than two ferroic order parameters can coexist and are coupled, by using an external magnetic field, or vice versa, has drawn great attention due to its scientific interest and technological importance [1-3]. For the change of polarization (magnetoelectric effect) and dielectric constant (magnetodielectric effect) upon a magnetic field, there

T. Bonaedy $\cdot$ Y.S. Koo $\cdot$ K.D. Sung $\cdot$ K.M. Song $\cdot$ N. Hur $\cdot$

J.H. Jung $(\varangle)$

Department of Physics, Inha University, Incheon 402-751, South

Korea

e-mail: jhjung@inha.ac.kr have been lots of theoretical and experimental works on the detailed mechanisms, such as the strong coupling between piezoelectricity and magnetostriction $[4,5]$.

On the other hand, recently, Catalan reported that the magnetodielectric effect can occur through a combination of the Maxwell-Wagner effect and magnetoresistance, called the resistive magnetodielectric effect, in single crystals with large charge depleted interfacial layers, superlattices, and polycrystalline samples, even without the magnetoelectric coupling [6, 7]. Such an extrinsic origin of the magnetodielectric effect could be practically useful due to the scarcity of magnetoelectric multiferroic single crystals [8, 9]. By choosing a material with large magnetoresistance, several experimental works have reported a large magnetodielectric effect near room temperature $[10,11]$. However, there are few experimental works on the effect of the grain boundary in the Maxwell-Wagner model for the resistive magnetodielectric property in polycrystalline samples.

In this paper, we investigate the effect of the grain boundary on the resistive magnetodielectric property of polycrystalline $\gamma-\mathrm{Fe}_{2} \mathrm{O}_{3}$ samples through impedance spectroscopy measurements. Although the value of magnetoresistance is quite similar, we have found that the value of the magnetodielectric effect and the resonance frequency for the maximum magnetodielectric effect depend on the resistance ratio between the grain and the grain boundary of the samples. This work suggests that the control of the grain boundary is one of the simplest ways to maximize the resistive magnetodielectric effect for possible applications.

\section{Experiments}

High-quality polycrystalline $\gamma-\mathrm{Fe}_{2} \mathrm{O}_{3}$ samples were prepared through the conventional sonochemical method and 
subsequent heat treatment, as described elsewhere [12]. The obtained powders were hydrostatically pressed $(\sim 200 \mathrm{MPa})$ into a disk shape and fired at 200 and $250^{\circ} \mathrm{C}$ for $24 \mathrm{~h}$ in atmosphere. Hereafter, we call $\gamma-\mathrm{Fe}_{2} \mathrm{O}_{3}$ fired at 200 and $250^{\circ} \mathrm{C} \gamma-\mathrm{Fe}_{2} \mathrm{O}_{3}$ (I) and $\gamma-\mathrm{Fe}_{2} \mathrm{O}_{3}$ (II), respectively. By using $\mathrm{X}$-ray diffraction and optical absorption measurements, we have confirmed the single phase of both samples. (Below $150^{\circ} \mathrm{C}$ and above $350^{\circ} \mathrm{C}$ of the firing temperature, we could find some $\mathrm{Fe}_{3} \mathrm{O}_{4}$ and $\alpha-\mathrm{Fe}_{2} \mathrm{O}_{3}$ impurity phases, respectively.) Based on Scherrer's formula for the X-ray diffraction (311) peak and independent transmission electron microscope measurements, we found that the grain sizes of both $\gamma-\mathrm{Fe}_{2} \mathrm{O}_{3}$ (I) and $\gamma-\mathrm{Fe}_{2} \mathrm{O}_{3}$ (II) were quite similar at $\sim 25 \mathrm{~nm}$.

The complex dielectric constant $\left(\widetilde{\varepsilon}=\varepsilon_{1}+\mathrm{i} \varepsilon_{2}\right)$ and impedance $\left(\widetilde{Z}=Z_{1}+\mathrm{i} Z_{2}\right)$ were obtained by an LCR meter in the frequency range of $20 \mathrm{~Hz}$ to $1 \mathrm{MHz}$ and an applied voltage of $1 \mathrm{~V}$. For these measurements, the samples were cut into the form of a thin plate with a thickness of $\sim 1 \mathrm{~mm}$. Silver electrodes with an area of $\sim 14 \mathrm{~mm}^{2}$ were evaporated on both sides of the sample. By using a conventional fourprobe method, direct current resistivity was obtained. For magnetoresistance and magnetodielectric measurements, we applied an external magnetic field up to $9 \mathrm{~T}$, by a superconducting magnet. Irrespective of a separate set of $\gamma-\mathrm{Fe}_{2} \mathrm{O}_{3}$ (I) and $\gamma-\mathrm{Fe}_{2} \mathrm{O}_{3}$ (II), we obtained quite reproducible experimental data shown below.

\section{Results and discussion}

Figure 1 shows the frequency-dependent dielectric constant $\left(\varepsilon_{1}\right)$ of $\gamma-\mathrm{Fe}_{2} \mathrm{O}_{3}$ (I) (a solid line) and $\gamma-\mathrm{Fe}_{2} \mathrm{O}_{3}$ (II) (a dashed line) at $293 \mathrm{~K}$. The values of $\varepsilon_{1}$ show strong frequency as well as temperature (see the inset of Fig. 1) dependences.

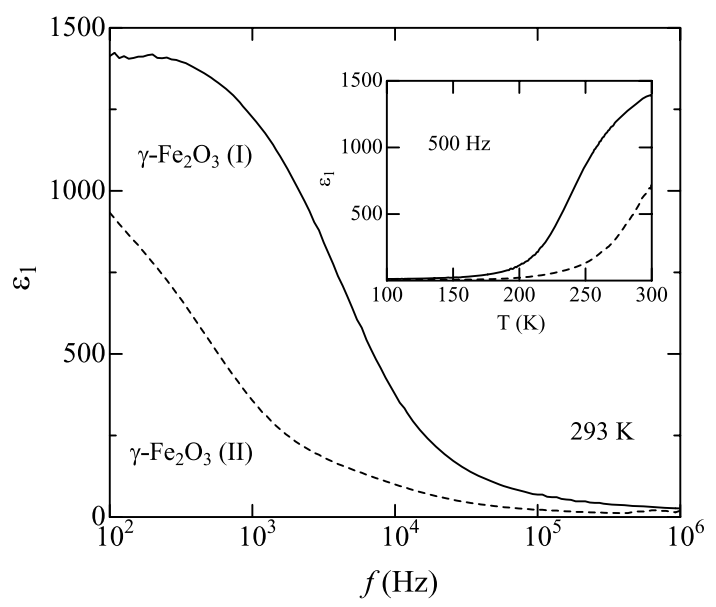

Fig. 1 The frequency-dependent dielectric constants of $\gamma-\mathrm{Fe}_{2} \mathrm{O}_{3}$ (I) (solid line) and $\gamma-\mathrm{Fe}_{2} \mathrm{O}_{3}$ (II) (dashed line) at $293 \mathrm{~K}$. In the inset, temperature-dependent dielectric constants at $500 \mathrm{~Hz}$ are also shown
For example, the ratio of $\varepsilon_{1}$ between $10^{2}$ and $10^{6} \mathrm{~Hz}$ is estimated to be $\sim 60$ for a $\gamma-\mathrm{Fe}_{2} \mathrm{O}_{3}$ (I) sample. While the values of $\varepsilon_{1}$ at low frequencies $\left(<10^{4} \mathrm{~Hz}\right)$ and high temperatures (>200 K) are quite different for the two samples, these values are quite similar at high frequencies and low temperatures.

Such a large value of $\varepsilon_{1}$ even in non-ferroelectric $\gamma-\mathrm{Fe}_{2} \mathrm{O}_{3}$, and its strong frequency as well as temperature dependences, have been explained by a possible Maxwell-Wagner two-capacitor model $[12,13]$ as similar to $\mathrm{CaCu}_{3} \mathrm{Ti}_{4} \mathrm{O}_{12}$ [14]. Due to the polycrystalline nature of our $\gamma-\mathrm{Fe}_{2} \mathrm{O}_{3}$, the resistance and capacitance of the grain and grain boundary could be different and depend on the sample preparation temperature. According to the Maxwell-Wagner model [13], the complex dielectric constant can be written as

$$
\begin{aligned}
& \varepsilon_{1}=\frac{1}{C_{0}\left(R_{\mathrm{G}}+R_{\mathrm{GB}}\right)} \frac{\tau_{\mathrm{G}}+\tau_{\mathrm{GB}}-\tau+\omega^{2} \tau_{\mathrm{G}} \tau_{\mathrm{GB}} \tau}{1+\omega^{2} \tau^{2}}, \\
& \varepsilon_{2}=\frac{1}{\omega C_{0}\left(R_{\mathrm{G}}+R_{\mathrm{GB}}\right)} \frac{1-\omega^{2} \tau_{\mathrm{G}} \tau_{\mathrm{GB}}+\omega^{2} \tau\left(\tau_{\mathrm{G}}+\tau_{\mathrm{GB}}\right)}{1+\omega^{2} \tau^{2}}
\end{aligned}
$$

where sub-indexes $G$ and GB refer to the grain and the grain boundary, respectively. Also, $R=$ resistance, $C=$ capacitance, $\omega=$ ac frequency, $\tau_{\mathrm{G}}=R_{\mathrm{G}} C_{\mathrm{G}}, \tau_{\mathrm{GB}}=R_{\mathrm{GB}} C_{\mathrm{GB}}$, $\tau=\left(\tau_{\mathrm{G}} R_{\mathrm{GB}}+\tau_{\mathrm{GB}} R_{\mathrm{G}}\right) /\left(R_{\mathrm{G}}+R_{\mathrm{GB}}\right)$, and $C_{0}=$ capacitance of vacuum. Especially, $\varepsilon_{1}$ can be approximated at low and high frequencies, respectively, as

$$
\begin{aligned}
& \varepsilon_{1}(\omega \rightarrow 0)=\frac{R_{\mathrm{G}}^{2} C_{\mathrm{G}}+R_{\mathrm{GB}}^{2} C_{\mathrm{GB}}}{C_{0}\left(R_{\mathrm{G}}+R_{\mathrm{GB}}\right)^{2}}, \\
& \varepsilon_{1}(\omega \rightarrow \infty)=\frac{C_{\mathrm{G}} C_{\mathrm{GB}}}{C_{0}\left(C_{\mathrm{G}}+C_{\mathrm{GB}}\right)} .
\end{aligned}
$$

From (3), it is quite clear that the dielectric constant at low frequency should depend on $R_{\mathrm{G}}$ and $R_{\mathrm{GB}}$, while that at high frequency is just the sum of $C_{\mathrm{G}}$ and $C_{\mathrm{GB}}$ in parallel. Therefore, the dielectric constant of the two $\gamma-\mathrm{Fe}_{2} \mathrm{O}_{3}$ samples at low frequency could be different, depending on the values of $R_{\mathrm{G}}$ and $R_{\mathrm{GB}}$ even with the same values of $C_{\mathrm{G}}$ and $C_{\mathrm{GB}}$.

To estimate the values of $R_{\mathrm{G}}, R_{\mathrm{GB}}, C_{\mathrm{G}}$, and $C_{\mathrm{GB}}$ of our $\gamma-\mathrm{Fe}_{2} \mathrm{O}_{3}$, we have performed an impedance spectroscopy analysis. ${ }^{1}$ Figure 2 shows the complex impedance plot $\left(Z_{2}\right.$ vs $Z_{1}$ ) of $\gamma-\mathrm{Fe}_{2} \mathrm{O}_{3}$ (I) (open circles) and $\gamma-\mathrm{Fe}_{2} \mathrm{O}_{3}$ (II) (open squares) at $293 \mathrm{~K}$. For the frequency range of our measurement, i.e. $20 \mathrm{~Hz}$ to $1 \mathrm{MHz}$, the impedance plot clearly shows the two semicircles for both samples. The value of $Z_{1}$ at $20 \mathrm{~Hz}$ for $\gamma-\mathrm{Fe}_{2} \mathrm{O}_{3}$ (II) is around two times larger than that

\footnotetext{
${ }^{1}$ Complex impedance and complex dielectric constant are interrelated as $\widetilde{Z}=1 /\left(\mathrm{i} \omega C_{0} \widetilde{\varepsilon}\right)$.
} 


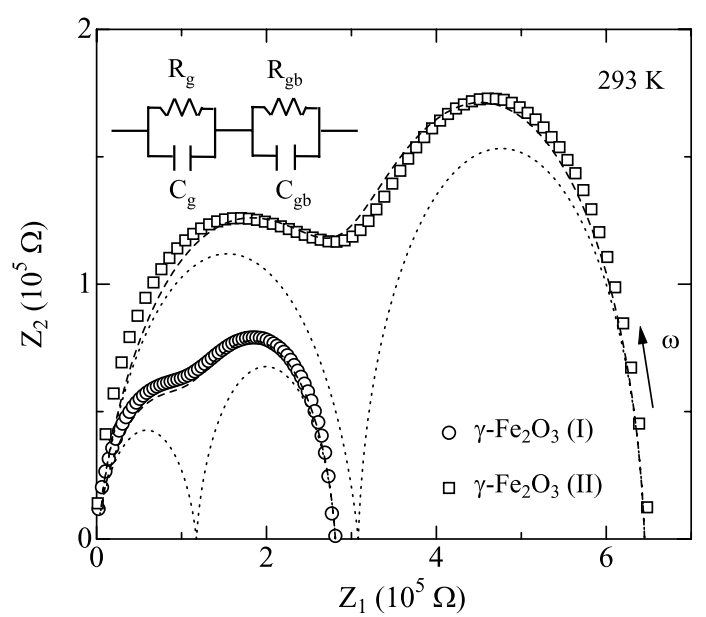

Fig. 2 Complex impedance spectroscopy plots of $\gamma-\mathrm{Fe}_{2} \mathrm{O}_{3}$ (I) (open circles) and $\gamma-\mathrm{Fe}_{2} \mathrm{O}_{3}$ (II) (open squares) at $293 \mathrm{~K}$. Note that the scales of $Z_{1}$ and $Z_{2}$ axes are different. The dotted and dashed lines represent the contributions of each semicircle and the sum of two semicircles, respectively. In the inset, an equivalent circuit is schematically shown

for $\gamma-\mathrm{Fe}_{2} \mathrm{O}_{3}(\mathrm{I})$, and that at $10^{6} \mathrm{~Hz}$ is nearly zero for both samples.

For the Maxwell-Wagner model, the impedance of a material has been modeled by two leaky capacitors in series, as schematically shown in the inset of Fig. 2. According to the general expression for impedance analysis [15], the semicircle on the left (at high frequency) is assigned as the contribution of the grain, while the semicircle on the right (at low frequency) is the result of the grain boundary. Also, the intercept of the semicircles on the real axis gives the resistance of the corresponding component contributing to the impedance of the sample.

Based on this approach, we fit the experimental data and obtain the values of $R_{\mathrm{G}}\left(R_{\mathrm{GB}}\right)$ as $\sim 1.17 \mathrm{M} \Omega(\sim 1.64 \mathrm{M} \Omega)$ for $\gamma-\mathrm{Fe}_{2} \mathrm{O}_{3}$ (I) and $\sim 3.03 \mathrm{M} \Omega(\sim 3.42 \mathrm{M} \Omega)$ for $\gamma-\mathrm{Fe}_{2} \mathrm{O}_{3}$ (II). Note that the centers of the two semicircles of our $\gamma-\mathrm{Fe}_{2} \mathrm{O}_{3}$ are on axes down below the real $Z_{1}$ axis. Therefore, instead of the relation of $\omega R_{\mathrm{G}} C_{\mathrm{G}}=\omega R_{\mathrm{GB}} C_{\mathrm{GB}}=1$ for the peak positions of two semicircles, we obtain the values of $C_{\mathrm{G}}$ and $C_{\mathrm{GB}}$ from the values of $R_{\mathrm{G}}$ and $R_{\mathrm{GB}}$ in (1), and also by fitting the magnetodielectric curves (see below), i.e. $C_{\mathrm{G}}\left(C_{\mathrm{GB}}\right)$ as $\sim 4.6 \mathrm{pF}(\sim 6 \mathrm{pF})$ for both samples. (For a separate set of $\gamma-\mathrm{Fe}_{2} \mathrm{O}_{3}$ (I) and $\gamma-\mathrm{Fe}_{2} \mathrm{O}_{3}$ (II), we have obtained nearly the same values of $R_{\mathrm{G}}, R_{\mathrm{GB}}, C_{\mathrm{G}}$, and $C_{\mathrm{GB}}$ within $5 \%$ error bar.)

Figures $3 \mathrm{a}$ and $\mathrm{b}$ show magnetodielectric $\left(\Delta \varepsilon_{1} / \varepsilon_{1}=\right.$ $\left.\left\{\varepsilon_{1}(H)-\varepsilon_{1}(0)\right\} / \varepsilon_{1}(0)\right)$ and magnetoresistance $(\Delta R / R=$ $\{R(H)-R(0)\} / R(0))$ curves, respectively, for $\gamma-\mathrm{Fe}_{2} \mathrm{O}_{3}$ (I) (open circles) and $\gamma-\mathrm{Fe}_{2} \mathrm{O}_{3}$ (II) (open squares) at $293 \mathrm{~K}$. While the resistance decreases with the increase of the external magnetic field, i.e. negative magnetoresistance, the dielectric constant increases with the increase of the external magnetic field, i.e. positive magnetodielectric effect. How-

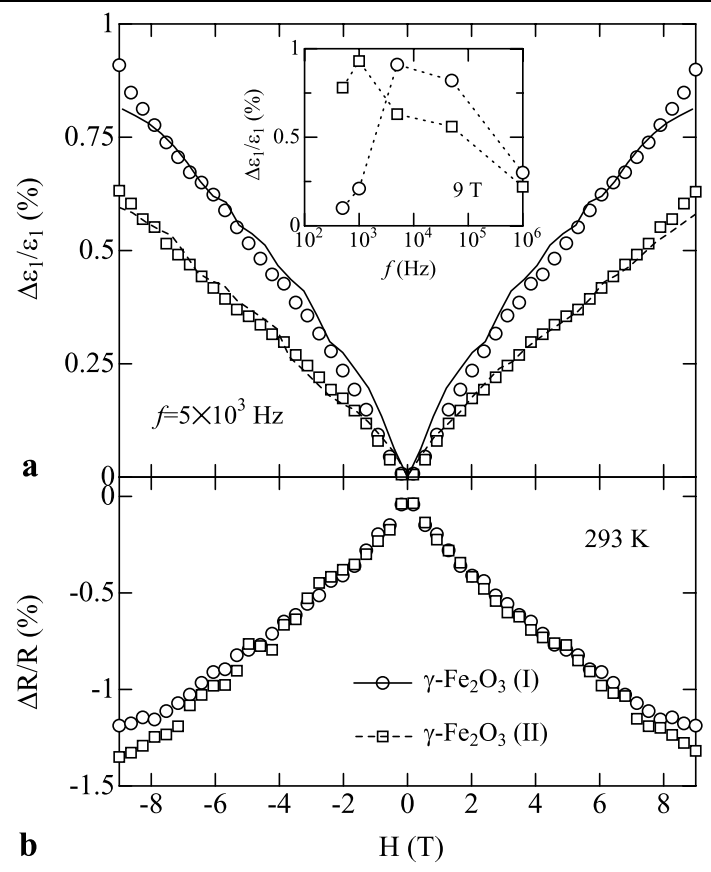

Fig. 3 (a) Measured (open dots) and calculated (solid lines) magnetodielectric curves at $5 \times 10^{3} \mathrm{~Hz}$ and $(\mathbf{b})$ magnetoresistance of $\gamma-\mathrm{Fe}_{2} \mathrm{O}_{3}$ (I) (open circles) and $\gamma-\mathrm{Fe}_{2} \mathrm{O}_{3}$ (II) (open squares) at $293 \mathrm{~K}$. In the inset of (a), we also show the values of the magnetodielectric effect at $9 \mathrm{~T}$ for selected frequencies

ever, one may clearly notice that while the values of the magnetoresistance are similar for the two samples, i.e. $\sim-1.3 \%$ at $9 \mathrm{~T}$, those of the magnetodielectric effect are different, i.e. $\sim 0.91 \%$ for $\gamma-\mathrm{Fe}_{2} \mathrm{O}_{3}$ (I) and $\sim 0.63 \%$ for $\gamma-\mathrm{Fe}_{2} \mathrm{O}_{3}$ (II) at $5 \times 10^{3} \mathrm{~Hz}$. As shown in the inset of Fig. 3a, the magnetodielectric effect at $9 \mathrm{~T}$ seems to be maximized near $5 \times 10^{3} \mathrm{~Hz}$ for $\gamma-\mathrm{Fe}_{2} \mathrm{O}_{3}$ (I), while it is maximized near $1 \times 10^{3} \mathrm{~Hz}$ for $\gamma-\mathrm{Fe}_{2} \mathrm{O}_{3}$ (II).

The main reason for the deviation of the magnetodielectric curves for the two $\gamma-\mathrm{Fe}_{2} \mathrm{O}_{3}$ samples, even with the quite similar values of magnetoresistance and capacitance, should come from the different values of the resistance ratio between the grain and the grain boundary, i.e. $R_{\mathrm{GB}} / R_{\mathrm{G}} \sim 1.40$ for $\gamma-\mathrm{Fe}_{2} \mathrm{O}_{3}$ (I) and $R_{\mathrm{GB}} / R_{\mathrm{G}} \sim 1.13$ for $\gamma-\mathrm{Fe}_{2} \mathrm{O}_{3}$ (II). According to (1), the value of $\Delta \varepsilon_{1} / \varepsilon_{1}$ should depend on the values of $\Delta R / R, R_{\mathrm{GB}} / R_{\mathrm{G}}$, and $C_{\mathrm{GB}} / C_{\mathrm{G}}$. To directly show the effect of $R_{\mathrm{GB}} / R_{\mathrm{G}}$ in our $\gamma-\mathrm{Fe}_{2} \mathrm{O}_{3}$, we calculated the magnetodielectric curves by inserting the experimentally obtained $\Delta R / R,{ }^{2} R_{\mathrm{GB}} / R_{\mathrm{G}}$, and $C_{\mathrm{GB}} / C_{\mathrm{G}}$ in (1). As shown clearly in Fig. 3a, the calculated $\Delta \varepsilon_{1} / \varepsilon_{1}$ (a solid line for $\gamma-\mathrm{Fe}_{2} \mathrm{O}_{3}$ (I) and a dashed line for $\gamma-\mathrm{Fe}_{2} \mathrm{O}_{3}$ (II)) fit the experimental $\Delta \varepsilon_{1} / \varepsilon_{1}$ curves well for both samples.

\footnotetext{
${ }^{2}$ We assumed that magnetoresistance should occur only in the grain, since it is difficult for tunneling magnetoresistance through the grain boundary to occur at high temperatures like $293 \mathrm{~K}$.
} 
Enhancement of the magnetodielectric effect through the increase of the resistance ratio between the grain and grain boundary in $\gamma-\mathrm{Fe}_{2} \mathrm{O}_{3}$ might imply the possible tunability of the resistive magnetodielectric effect through grain boundary control. It is well known that the resistance of the grain boundary is easily controlled by changing the annealing temperature and/or environment [16]. Therefore, we may infer that polycrystalline ferromagnetic insulators with large resistance ratio between the grain and the grain boundary, besides large magnetoresistance, could be one of the good candidates for the possible device application by using the resistive magnetodielectric effect.

\section{Summary}

In summary, we showed that the resistance ratio between the grain and the grain boundary is one of the key factors to control the magnetodielectric effect in polycrystalline $\gamma-\mathrm{Fe}_{2} \mathrm{O}_{3}$ samples through impedance spectroscopy measurements. Our result suggests that the control of the grain boundary through the annealing environment and/or temperature should be quite useful for possible magnetodielectric devices.

Acknowledgements This work was supported by the Korea Research Foundation Grant funded by the Korean Government (MOEHRD) (KRF-2005-070-C00053) and (KRF-2006-331-C00092).

\section{References}

1. T. Kimura, T. Goto, H. Shintani, K. Ishizaka, T. Arima, Y. Tokura, Nature (Lond.) 426, 55 (2003)
2. N. Hur, S. Park, P.A. Sharma, J.S. Ahn, S. Guha, S.-W. Cheong, Nature (Lond.) 429, 392 (2004)

3. M. Gajek, M. Bibes, S. Fusil, K. Bouzehouane, J. Fontcuberta, A. Barthélémy, A. Fert, Nat. Mater. 6, 296 (2007)

4. H. Zheng, J. Wang, S.E. Lofland, Z. Ma, L. Mohaddes-Ardabili, T. Zhao, L. Salamanca-Riba, S.R. Shinde, S.B. Ogale, F. Bai, D. Viehland, Y. Jia, D.G. Schlom, M. Wuttig, A. Roytburd, R. Ramesh, Science 303, 661 (2004)

5. C.-W. Nan, G. Liu, Y.-H. Lin, H. Chen, Phys. Rev. Lett. 94, 197203 (2005)

6. G. Catalan, Appl. Phys. Lett. 88, 102902 (2006)

7. G. Catalan, J.F. Scott, Nature (Lond.) 448, E4 (2007)

8. G. Lawes, R. Tackett, O. Masala, B. Adhikary, R. Naik, R. Seshadri, Appl. Phys. Lett. 88, 242903 (2006)

9. Y.S. Koo, T. Bonaedy, K.D. Sung, J.H. Jung, J.B. Yoon, Y.H. Jo, M.H. Jung, H.J. Lee, T.Y. Koo, Y.H. Jeong, Appl. Phys. Lett. 91, 212903 (2007)

10. J. Rivas, J. Mira, B. Rivas-Murias, A. Fondado, J. Dec, W. Kleemann, M.A. Señarís-Rodríguez, Appl. Phys. Lett. 88, 242906 (2006)

11. J. Cao, R.C. Rai, S. Brown, J.L. Musfeldt, R. Tackett, G. Lawes, Y.J. Wang, X. Wei, M. Apostu, R. Suryanarayanan, A. Revcolevschi, Appl. Phys. Lett. 91, 021913 (2007)

12. T. Bonaedy, Y.S. Koo, K.D. Sung, J.H. Jung, Appl. Phys. Lett. 91, 132901 (2007)

13. A. von Hippel, Dielectrics and Waves (Artech House, London, 1995)

14. D.C. Sinclair, T.B. Adams, F.D. Morrison, A.R. West, Appl. Phys. Lett. 80, 2153 (2002)

15. E. Barsoukov, J.R. Macdonald, Impedance Spectroscopy (Wiley, New York, 2005)

16. A.J. Moulson, J.M. Herbert, Electroceramics: Materials, Properties and Applications (Chapman and Hall, London, 1990) 